\section{Why we must reduce dental prescription of antibiotics: European Union Antibiotic Awareness Day}

\author{
M. A. 0. Lewis ${ }^{1}$
}

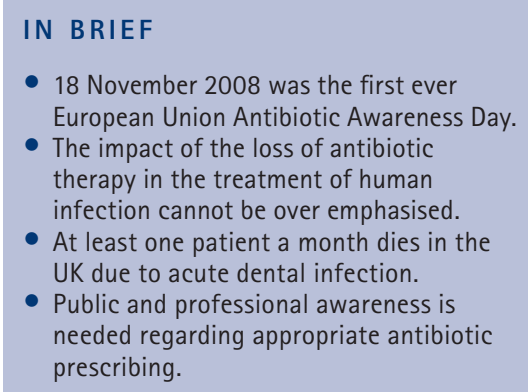

The prescription of antibiotics in dentistry is relatively small but nonetheless significant. With the emergence of bacterial species resistant to antibiotics there is a need to become vigilant about their prescription and with this, an urgent requirement for both professional and public understanding of the appropriate use of this life-saving component of treatment.

Since the discovery of penicillin, the first antibiotic, by Alexander Fleming in 1928 and its subsequent introduction to clinical practice by Florey in 1940, antibiotics have been used extensively in dentistry. Interestingly from a dental perspective, one of the first reports of the clinical effectiveness of penicillin involved the successful treatment of a patient with cellulitis and angular cheilitis. However, while many bacterial species were initially found to be sensitive to penicillin, there has been a gradual and sustained emergence of resistant strains. Similar patterns of reducing susceptibility have been observed for all types of antibiotic that have subsequently been developed during the past 50 years. We have now entered an era where some bacterial species are resistant to the full range of antibiotics presently available. Perhaps the most widely known example of extensive resistance is methicillin-resistant Staphylococcus aureus (MRSA), also known commonly as a 'superbug'. MRSA is rarely out of the national press due to its association

${ }^{1}$ Associate Dean of Postgraduate Studies/Professor of Oral Medicine, School of Dentistry, Cardiff University, Heath Park, Cardiff, CF14 4XY; Dental Member of the Advisory Committee for Antimicrobial

Resistance and Hospital Associated Infection

Correspondence to: Professor Michael A. O. Lewis Email: LewisMA0@cardiff.ac.uk

\section{Refereed Paper}

Accepted 1 November 2008

DOI: 10.1038/sj.bdj.2008.984

${ }^{\circledR}$ British Dental Journal 2008; 205: 537-538 with severe infection and death in hospitalised patients. The impact of the loss of antibiotics as effective agents in the treatment of human infection cannot be over-emphasised and this truly represents a potential medical disaster.

\section{The emergence of antibiotic resistance}

It is from the background of the worldwide emergence of antibiotic resistance that the European Centre for Disease Prevention and Control (ECDC) marked Tuesday 18 November 2008 as the first ever European Union Antibiotic Awareness Day in an attempt to improve the use of antibiotic therapy within the community and the healthcare professions. Of the 40 million oral courses of antibiotics dispensed in England each year, a significant proportion (over three million) is prescribed by dentists. Antibiotics account for the vast majority of medicines prescribed by dentists, with analgesics, antifungals and antiviral agents accounting for a small proportion of prescriptions. In 2004, a survey of over 6,000 general dental practitioners in the UK revealed that $40 \%$ of dentists were prescribing antibiotics on at least three occasions every week. The research also revealed that 15\% of the dentists prescribed antibiotics on a daily basis. However, it is increasingly being accepted that such prescribing habits are often either inappropriate or unnecessary. A study of 500 patients presenting at a secondary care dental emergency unit demonstrated that 57\% had been provided with an antibiotic in the absence of a recognised clinical indication for such medication. Furthermore, clinical audits have shown that prescribing an antibiotic makes no difference to the outcome of treatment of acute dental infection if drainage is established. Unfortunately it is well recognised that patients are also routinely provided with one or more antibiotics in these circumstances, despite the availability of range of guidelines advising against this practice.

The spectrum of antibiotics used in dentistry in the United Kingdom is fortunately small, with amoxicillin and phenoxymethylpenicillin having been the two drugs most frequently prescribed during the past 25 years. There is now clear evidence linking the dental prescribing of these antimicrobial agents to the emergence of penicillin resistance in the community. Perhaps of greater concern are the susceptibility surveillance studies that have demonstrated that the incidence of penicillin resistance within the microflora of acute dental infections has risen from a relatively low level of 5\% of abscesses to more than 55\% of cases in the past decade. Coincidently at the same time, maxillofacial surgery units are reporting an increase in the number of severe cases of dental infection requiring admission for management. The figures for England show that admissions for 'drainage of an abscess related to tooth' has doubled 
from just under 800 in 1998 to almost 1,600 in 2006. Although it has been postulated that this increase may in part reflect reduced access to primary dental care, it may also reflect the emergence of resistance to first line antibiotic therapy. Unfortunately, culture and sensitivity of pus specimens from dental infections is not routinely undertaken in the vast majority of hospitals and this has severely limited the availability of antimicrobial resistance data. The majority of dental healthcare workers are likely to be unaware that at least one patient a month dies in the United Kingdom due to unsuccessful management of acute dental infection.

\section{Reducing dental prescriptions}

Two of the main factors associated with development of resistance are duration of therapy and dose of drug. Individuals within the community can harbour resistant bacterial strains for up to three months following antibiotic usage and as such, represent a high level of resistance within the general population. If the number of prescriptions issued in dentistry could be reduced then the selection of resistant strains should occur less frequently. It has recently been shown that reducing antibiotic prescribing for urinary tract infections at the general medical practice level resulted in a reduced incidence of resistance in the local community. This observation is extremely important since it does demonstrate to individual practitioners that modifying their prescribing habits can influence patterns of resistance. The same beneficial effect would probably be observed for dental practitioners if they reviewed their prescribing for dental conditions.

It is important that not only the dental profession but the general public understand the importance of restricting the use of antibiotics to those true cases of severe infection that require them. Patients have become accustomed to being given an antibiotic for a range of medical complaints. Unfortunately, patients presenting at dental surgeries also routinely expect an antibiotics for the treatment of 'toothache'. It is difficult to explain to the patient, and occasionally their relatives, that dental pain is an inflammatory condition that is appropriately managed by use of analgesics and local measures and not a bacterial infection that requires provision of an antibiotic. The same problematic situation is frequently encountered in general medical practice, where patients with dental pain request the provision of an antibiotic.

\section{Convincing patients and professionals}

In the United Kingdom, the Advisory Committee for Antimicrobial Resistance and Hospital Associated Infection (ARHAI) is working to help encourage the development of more formal systems to monitor both hospital and community prescribing. Equally important would be the collection of information that demonstrates the successful clinical outcome of the "no antibiotic prescribing' option. All efforts must be made to convince patients, families, dentists and doctors that it is both appropriate and safe to treat toothache and the majority of acute dental conditions without the use of an antibiotic. This will require investment in auditing systems to show that low prescribing rates are best practice. There is no doubt that achieving low prescribing rates will be a considerable challenge to the profession due to the pressure exhibited by patients expecting antibiotic therapy.

In summary, there is an urgent need to raise public and professional awareness regarding the risks of antibiotic use not only in dentistry but all aspects of medical care. The establishment of an EU Antibiotic Day is an excellent start and it is hoped that this opinion article will inspire dental professionals to think about the necessity to prescribe an antibiotic and also, if they do write a prescription, the implications of their actions. If the profession and public respond responsibly to the issues surrounding antibiotic prescribing now, it may be possible to preserve the usefulness of these drugs for clinical situations where they are truly indicated. Ignoring these problems will undoubtedly result in an increase in incidence of untreatable dental infections and most importantly, death of patients from dental disease in the future. 